\title{
Targeted silencing of TEM8 suppresses non-small cell lung cancer tumor growth via the ERK/Bcl-2 signaling pathway
}

\author{
QUAN GONG $^{1 *}$, JING DENG $^{1 *}$, LIJUAN ZHANG $^{1}$, CHUNYAN ZHOU $^{1}$, \\ CHAOJIANG FU ${ }^{2}$, XICAI WANG ${ }^{3}$ and LI ZHUANG $^{1}$ \\ Departments of ${ }^{1}$ Palliative Medicine,${ }^{2}$ Critical Care Medicine and ${ }^{3}$ Tumor Research Institute, \\ Third Affiliated Hospital of Kunming Medical University, Kunming, Yunnan 650118, P.R. China
}

Received January 10, 2021; Accepted May 24, 2021

DOI: $10.3892 / \mathrm{mmr} .2021 .12234$

\begin{abstract}
Non-small cell lung cancer (NSCLC) is one of the most common malignancies with high rates of mortality. Although great progress has been made with the development of novel immunotherapies and targeted therapeutic strategies, the 5-year total survival rate of lung cancer has remained unchanged over the past few decades. Therefore, more effective therapeutics are urgently needed. Tumor endothelial marker 8 (TEM8) is an integrin-like cell surface transmembrane protein that has been demonstrated to be upregulated in numerous cancer types and previously showed promise for targeted cancer therapy. However, the role of TEM8 in NSCLC remains poorly understood. The present study aimed to investigate the effects of silencing TEM8 on expression and regulation of extracellular signal-regulated kinase (ERK)1/2 signaling pathways in NSCLC. In the present study, a lentiviral vector that encoded a short hairpin RNA targeting TEM8 was designed and transfected into Xuanwei Lung Cancer (XWLC)-05 lung cancer cells to silence TEM8 expression. Male BALB/c-nu/nu mice were then given subcutaneous injections in the right dorsal flank with XWLC-05 cells. Microvessel density was measured using an anti-CD34 antibody. The mRNA and protein levels of ERK1/2 and Bcl-2 in XWLC-05 cells or xenograft tumor tissues were detected by reverse transcription-quantitative polymerase chain reaction and western blotting. TEM8 knockdown was found to significantly inhibit tumor growth and conferred an anti-angiogenic ability in vivo. Furthermore, TEM8 knockdown suppressed the expression of Bcl-2 mediated by ERK1/2 activity in XWLC-05 cells or tissues from mice with NSCLC. To conclude, these results suggest
\end{abstract}

Correspondence to: Professor Li Zhuang, Department of Palliative Medicine, Third Affiliated Hospital of Kunming Medical University, 519 Kunzhou Road, Kunming, Yunnan 650118, P.R. China

E-mail: kekzhuangli@163.com

${ }^{*}$ Contributed equally

Key words: tumor endothelial marker 8, non-small cell lung cancer, angiogenesis, ERK/Bcl-2 signaling pathway that the targeted silencing of TEM8 may serve as an effective method of treating NSCLC.

\section{Introduction}

Lung cancer is one of the most common malignant tumors and the main cause of cancer-associated mortality, with $>1.7$ million deaths reported worldwide in 2018 (1). Lung cancer can be broadly divided into two major categories based on its histological characteristics: Small cell lung cancer (SCLC) and non-small cell lung cancer (NSCLC) $(2,3)$. Compared with SCLC, NSCLC is the main type of lung cancer and accounts for $>84 \%$ of cases of lung cancer (4). The clinical efficacy of current therapeutic strategies of lung cancer is greatly dependent on the stage of disease, where poor outcomes are closely associated with diagnosis at advanced stages and propensity for metastasis (5). By contrast, good outcomes are associated with diagnosis at early stages (5). However, most patients with NSCLC are typically diagnosed at advanced or metastatic stages of the disease (6). Although major improvements have been made in the treatment of NSCLC with the development of targeted therapies and immunotherapy, the 5-year survival rate of patients with lung cancer has remained unchanged over the past number of decades (7). NSCLC is also the archetype of a genomics-driven malignancy, such as YES1 (a member of SRC family kinases) mutation, which showed a promising therapeutic target in lung cancer (8). However, YES1 status can only be used as a predictive biomarker for treatment in a selected subset of patients, which means that only a small proportion of patients with lung cancer can benefit from targeted therapy (8). Therefore, it is imperative to identify new druggable targets to enhance the efficacy of the currently available therapies and to reduce the mortality rate of lung cancer.

Tumor endothelial marker 8 (TEM8) is an integrin-like cell surface transmembrane protein that was first identified as a marker in the tumor endothelium in colorectal cancer by St Croix et al (9) in 2000. TEM8 has been found to be upregulated in the stroma of numerous solid tumors, including osteosarcoma (10), colorectal cancer (9), lung cancer (11), melanoma (12) and triple-negative breast cancer (13). This makes it an attractive target for studying any potentially novel anticancer therapeutics, due to its overexpression on the cell 
surface of solid tumors. Previous studies in human tumor xenografts have reported that either blocking or knocking down TEM8 expression can inhibit tumor growth $(14,15)$. In addition, previous studies have confirmed that TEM8 knockdown could inhibit the migration and invasion of Xuanwei Lung Cancer (XWLC)-05 cells in vitro (11). Since the XWLC-05 cell line has been proved to exhibit histological characteristics that are similar to those observed in NSCLC (16), the present study mainly focused on the effects of silencing TEM8 expression on NSCLC tumor growth in vivo.

Angiogenesis has been previously shown to be an important process in promoting tumor growth and metastasis (17), such that a number of anti-angiogenic agents (e.g., bevacizumab plus irinotecan, fluorouracil and leucovorin) are currently being applied clinically (18). TEM8 has been shown to be required for angiogenesis in melanoma, breast and colon cancer (15). However, TEM8 knockdown in mice was found not to affect the angiogenic process during normal development or wound healing (19). Taken together, these previously reported effects suggest that TEM8 is required for tumor angiogenesis, but not physiological angiogenesis. However, most of the known vascular endothelial markers are not only expressed in tumor tissues, but also in normal tissues, meaning that targeting these markers can lead to side effects $(19,20)$. Therefore, treatments that target TEM8 may inhibit tumor growth with less severe side effects. Nevertheless, little is known concerning the function of TEM8 in lung cancer in vivo.

In the present study, the effects of silencing TEM8 expression on tumor growth in a murine xenograft model of lung cancer was investigated. Specifically, the relationship between TEM8 expression and tumor angiogenesis was examined. Furthermore, the underlying signaling pathways that are activated following TEM8 knockdown was also measured. These findings may provide a novel theoretical basis for early lung cancer diagnosis and improving the outcome of targeted therapies.

\section{Materials and methods}

Cell lines. The XWLC-05 cell line was provided by Dr Wang Li, Radiotherapy Center of The Third Affiliated Hospital of Kunming Medical University (Kunming, China). The cells were cultured in RPMI-1640 medium (HyClone; Cytiva) supplemented with $10 \% \mathrm{FBS}$ (HyClone; Cytiva) at $37^{\circ} \mathrm{C}$ in a humidified incubator under $5 \% \mathrm{CO}_{2}$ atmosphere in T25 flasks. When the cell density reached $>90 \%$, cells were passaged at reasonable ratios for further study.

Experimental animals. A total of 18 male BALB/c-nu/nu mice

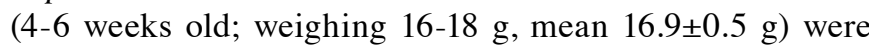
purchased from Beijing Vital River Laboratory Animal Technology Co., Ltd. All mice were randomly divided into three groups with six mice each. The mice were housed under a 12-h light/dark cycle at a constant temperature $\left(22-24^{\circ} \mathrm{C}\right)$ and humidity (40-70\%) in a specific pathogen-free environment with free access to food and water. The mice were acclimated to the housing conditions for 1 week before inoculation. All animal experiments were approved by the Committee on the Ethics of Animal Experiments of The Third Affiliated Hospital of Kunming Medical University (approval no. 16KY-LA00135).
Creation of the TEM8-short hairpin RNA (shRNA/sh) stable transfection cell line. The pGLVH1/GFP/Puro (PGLV3) vectors encoding TEM8-shRNA and negative control (NC) shRNA constructs were purchased from Shanghai GenePharma Co., Ltd. The lentivirus was synthesized by Shanghai GenePharma Co., Ltd. XWLC-05 cells were plated into a 96-well plate at a density of $5 \times 10^{4}$ cells/well and cultured in RPMI-1640 medium with $10 \% \mathrm{FBS}$ at $37^{\circ} \mathrm{C}$ overnight. The XWLC-05 cells were then infected with lentiviral or control virus (NC) in RPMI-1640 medium with $10 \%$ FBS and polybrene at a final concentration of $5 \mu \mathrm{g} / \mathrm{ml}$ according to the manufacturer's instructions. After $24 \mathrm{~h}$, the cells were washed three times with PBS and cultured in RPMI-1640 medium supplemented with $10 \% \mathrm{FBS}$ at $37^{\circ} \mathrm{C}$ for $72 \mathrm{~h}$. Efficiency of TEM 8 silencing was determined by reverse transcription-quantitative PCR (RT-qPCR) and western blotting under different conditions, namely untreated control XWLC-05 cells, shNC-transfected cells and shTEM8-transfected cells.

After $72 \mathrm{~h}$ transfection, the transfected or untransfected XWLC-05 cells were plated into a six-well plate at densities of $5 \times 10^{5}$ cells/well and fresh medium containing $2 \mu \mathrm{g} / \mathrm{ml}$ puromycin (Sigma-Aldrich; Merck KGaA) was added to select for stably transfected XWLC-05 cells. Subsequently, $2 \mu \mathrm{g} / \mathrm{ml}$ puromycin was used for maintenance of the stably transfected cell line. The stably transfected XWLC-05 cells were harvested and subsequently amplified.

In vivo tumorigenicity assays. All BALB/c-nu/nu mice were randomly divided into the following three groups ( $n=6 /$ group): i) Control group; ii) shNC group; and iii) shTEM8 group. XWLC-05 cells in the indicated groups were harvested and resuspended in PBS to a final concentration of $1 \times 10^{7}$ cells $/ \mathrm{ml}$. The mice were then given subcutaneous injections in the right dorsal flank with the prepared cells $\left(2 \times 10^{6}\right.$ cells/mouse), after which they were monitored every day. After the subcutaneous tumors become visible, the tumor volumes were measured with a vernier caliper once every 2 days and the tumor size was calculated using the following formula: (Length $\mathrm{x}$ width $\mathrm{x}$ height) $/ 2$. At 28 days post-injection, the maximum tumor size reached $\sim 1,200 \mathrm{~mm}^{3}$ in the control group all mice were sacrificed by cervical dislocation, and tumors were excised and weighed.

$R T$ - $q P C R$. Total RNA was extracted from tumor tissues or cells using TRIzol ${ }^{\circledR}$ reagent (Thermo Fisher Scientific, Inc.) according to the manufacturer's protocol. The quality and concentration of extracted RNA was measured using a UV/VIS spectrophotometer. First-strand cDNAs were synthesized using the High-Capacity cDNA Reverse Transcription kit (Thermo Fisher Scientific, Inc.) according to the manufacturer's protocol. qPCR was performed using an ABI 7500 Real-Time PCR detection system (Applied Biosciences; Thermo Fisher Scientific, Inc.) with SuperReal PreMix Plus (SYBR-Green) (Tiangen Biotech Co., Ltd.). The following qPCR thermocycling conditions were used: Pre-denaturation at $95^{\circ} \mathrm{C}$ for $10 \mathrm{~min}$; followed by 40 cycles of $95^{\circ} \mathrm{C}$ for $15 \mathrm{sec}$ and $60^{\circ} \mathrm{C}$ for $60 \mathrm{sec}$. The primers used for the RT-qPCR are shown in Table I, where GAPDH was used as the internal reference. The relative mRNA level was calculated using the $2^{-\Delta \Delta \mathrm{Cq}}$ method (21). Each experiment was repeated $\geq 3$ times. 
Table I. Primers used for reverse transcription-quantitative PCR.

\begin{tabular}{llc}
\hline Gene & \multicolumn{1}{c}{ Primer sequences $\left(5^{\prime} \rightarrow 3^{\prime}\right)$} & Production size $(\mathrm{bp})$ \\
\hline TEM8 & F: GCTGCACCACTGGAATGAAATCT & 153 \\
& R: AGGCCTTGACGGATTTGTTCTCT & 111 \\
ERK & F: TTACTGCGCTTCAGACATGAGA & \\
& R: ATCTGTTTCCATGAGGTCCTGT & F: TGTGTGTGGAGAGCGTCAAC \\
GAPDH & R: GGGCCGTACAGTTCCACAAA & 142 \\
& F: TCTCTGCTCCTCCTGTTCGA & 122
\end{tabular}

F, forward; R, reverse; TEM8, tumor endothelial marker 8; ERK, extracellular signal-regulated kinase.

Immunohistochemistry and immunofluorescence staining. Tumor tissues from each mouse were harvested at the end of the study, fixed with $4 \%$ paraformaldehyde for $12 \mathrm{~h}$ at room temperature and embedded in paraffin. The paraffin sections at a thicknesses of $4 \mu \mathrm{m}$ were prepared, dewaxed with xylene and rehydrated in ethanol, and antigen retrieval was performed in $0.01 \mathrm{~mol} / \mathrm{l}$ citrate buffer $(\mathrm{pH}$ 6.0) in a microwave oven for $20 \mathrm{~min}$ at $98-100^{\circ} \mathrm{C}$. The sections were then treated with $3 \%$ hydrogen peroxide methanol for $10 \mathrm{~min}$ at room temperature to block the endogenous peroxidase activity.

For immunohistochemistry, the sections were blocked with 1\% BSA (Beijing Solarbio Science \& Technology Co., Ltd.) in PBS containing 0.05\% Tween-20 (PBST) for $40 \mathrm{~min}$ at room temperature. The sections were incubated with the anti-TEM8 primary antibody (cat. no. ab21270; 1:200; Abcam) overnight at $4^{\circ} \mathrm{C}$. After washing three times with PBS, the sections were incubated with HRP-conjugated goat anti-rabbit (cat. no. ab6721; 1:500; Abcam) secondary antibodies at $37^{\circ} \mathrm{C}$ for $2 \mathrm{~h}$. The sections were then stained with diaminobenzidine (Beijing Solarbio Science \& Technology Co., Ltd.) at $37^{\circ} \mathrm{C}$ for 30 min after washing with PBS. The sections were counterstained with hematoxylin for $1 \mathrm{~min}$ at room temperature after the DAB reaction was stopped. The sections were then dehydrated in a graded ethanol series, transparentized with xylene, and mounted in neutral gum. The immunostained sections were examined using a fluorescence microscope at x200 magnification (Olympus Corporation).

For immunofluorescence, the sections were blocked with 1\% BSA (Beijing Solarbio Science \& Technology Co., Ltd.) in PBST for $40 \mathrm{~min}$ at room temperature. The sections were then incubated with the primary antibody against CD34 (cat. no. ab81289; 1:200; Abcam) overnight at $4^{\circ} \mathrm{C}$. After three washes with PBS, samples were incubated with the Alexa Fluor $^{\circledR}$ 594-conjugated secondary antibody (cat. no. ab150084; 1:200; Abcam) for $2 \mathrm{~h}$ at room temperature. After washing three times with PBS, cell nuclei were stained with 4',6-diamidino-2-phenylindole (cat. no. 36308ES11; Shanghai Yeasen Biotech Co., Ltd.) solution, and stored in the dark for $5 \mathrm{~min}$ at room temperature. The sections were imaged by fluorescence microscopy at x200 magnification (Olympus Corporation).

To determine the microvessel density (MVD) values, CD34-stained MVD was enumerated using the Weidner method (22). The positive microvessel quantity stained with CD34 in five different visual fields was counted under a light microscope (magnification, $\mathrm{x} 200$ ) to calculate the average as the value of MVD.

Hematoxylin and eosin $(H \& E)$ staining. For $H \& E$ staining, the sections were dewaxed in xylene and rehydrated in a graded alcohol series. Then, the sections were rinsed with tap water for $1 \mathrm{~min}$, immersed in hematoxylin (Beyotime Institute of Biotechnology) solution for $4 \mathrm{~min}$ at room temperature and rinsed with tap water for $30 \mathrm{sec}$. Sections were differentiated using a $1 \%$ acid ethanol solution for $2 \mathrm{sec}$ and rinsed with tap water for $5 \mathrm{~min}$. Then, the sections were stained with eosin (Beyotime Institute of Biotechnology) solution for $10 \mathrm{sec}$ at room temperature and rinsed with tap water, followed by dehydration with graded alcohol and clearing in xylene. Finally, the sections were dried and mounted with neutral gum seal. Sections were examined under a fluorescence microscope at x200 magnification (Olympus Corporation).

Western blotting. The tumor tissues or cells were lysed with ice-cold RIPA buffer (Beyotime Institute of Biotechnology) mixed with protease and phosphatase inhibitors (Beyotime Institute of Biotechnology). Following $30 \mathrm{~min}$ of lysis on ice, the tissues or cells were centrifuged at $12,000 \mathrm{x} \mathrm{g}$ for $12 \mathrm{~min}$ at $4^{\circ} \mathrm{C}$ and the supernatant was collected. Then, the protein concentration was quantified using a bicinchoninic acid protein assay kit (Beyotime Institute of Biotechnology). Protein samples $(\sim 50 \mu \mathrm{g})$ were separated via $10 \%$ SDS-PAGE, and then transferred onto polyvinylidene difluoride membranes (EMD Millipore). The membranes were blocked in $5 \%$ skimmed milk powder for $2 \mathrm{~h}$ at room temperature, and subsequently incubated overnight at $4{ }^{\circ} \mathrm{C}$ with the following primary antibodies: Anti-TEM8 (cat. no. ab21270; 1:1,000; Abcam), anti-extracellular signal-regulated kinase (ERK)1/2 (cat. no. 4695; 1:1,000; Cell Signaling Technology, Inc.), anti-Bcl-2 (cat. no. 4223; 1:1,000; Cell Signaling Technology, Inc.), anti-phosphorylated (p)-ERK1/2 (cat. no. 4370; 1:2,000; Cell Signaling Technology, Inc.) and anti-GAPDH (cat. no. 5174; 1:1,000; Cell Signaling Technology, Inc.). After washing three times with TBS containing $0.05 \%$ Tween-20 (TBST), the membranes were incubated with HRP-conjugated goat anti-rabbit secondary antibodies 

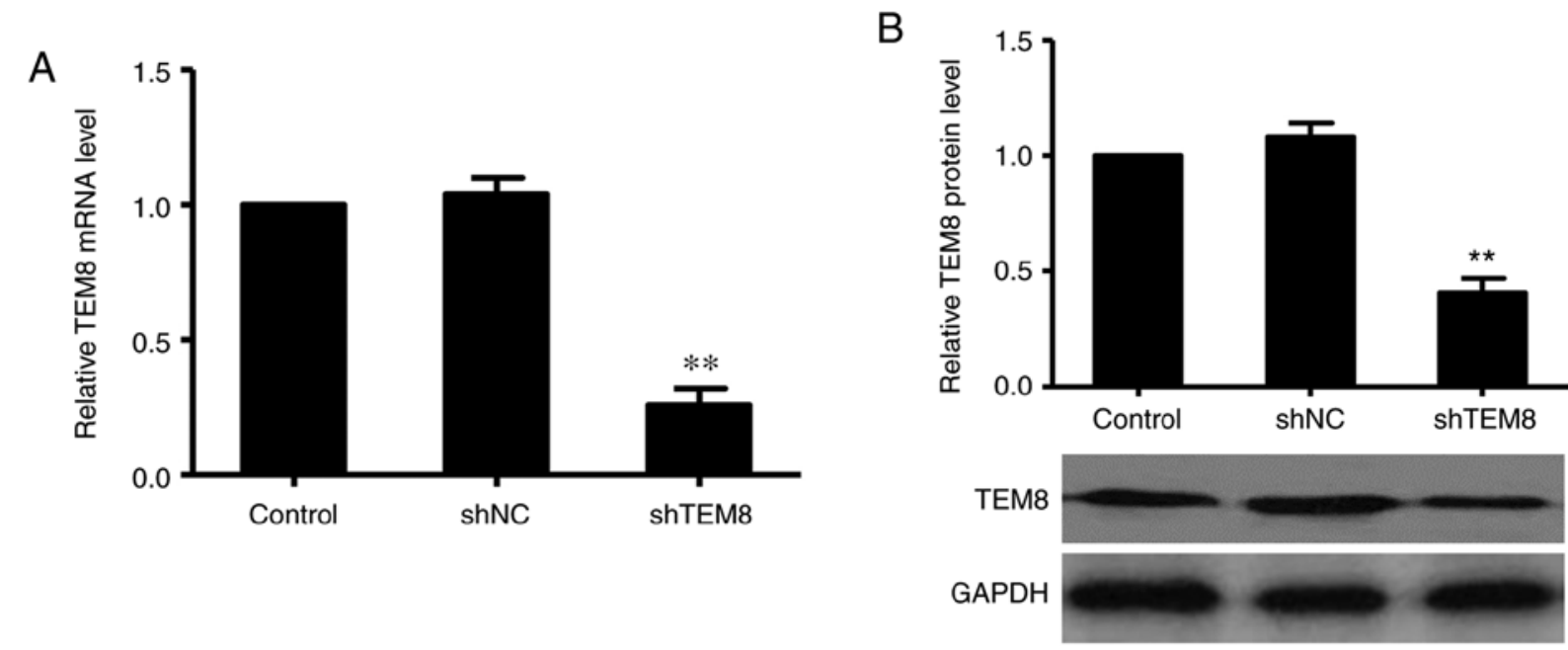

Figure 1. Expression of TEM8 in Xuanwei Lung Cancer-05 cells. (A) Expression of TEM8 mRNA in control, shNC and shTEM8 groups. (B) Expression of TEM8 protein in control, shNC and shTEM8 groups. ${ }^{* *} \mathrm{P}<0.01$ vs. Control group. TEM8, tumor endothelial marker 8 ; sh, short hairpin RNA; NC, negative control.

(cat. no. 7074; 1:5,000; Cell Signaling Technology, Inc.) for $2 \mathrm{~h}$ at room temperature. The immunoreactive bands were detected using the SuperSignal ${ }^{\mathrm{TM}}$ West Pico chemiluminescent substrate (Thermo Fisher Scientific, Inc.). The blots were semi-quantified using the Quantity One software version 4.62 (Bio-Rad Laboratories, Inc.) to analyze the gray value of each antibody band. The relative integrated optical density for TEM8, ERK1/2, p-ERK1/2 and Bcl-2 were normalized to that of GAPDH in the same sample.

Statistical analysis. Statistical differences were determined using a one-way ANOVA followed by a Tukey's post hoc test. All data were analyzed using SPSS software version 17.0 (SPSS, Inc.) and are expressed as the mean \pm SD. All experiments were repeated $\geq 3$ times. $\mathrm{P}<0.05$ was considered to indicate a statistically significant difference.

\section{Results}

Successful knockdown of TEM8 expression via transfection with shTEM8 in XWLC-05 cells. To evaluate the efficiency of gene silencing using shTEM8, XWLC-05 cells were transfected with either shTEM8 or shNC. After $72 \mathrm{~h}$ of transfection, TEM8 expression was measured using RT-qPCR and western blotting. The RT-qPCR results showed that TEM8 mRNA expression was significantly decreased in the shTEM8 group compared with that in the control group (Fig. 1A). Subsequently, western blotting revealed that TEM8 protein expression was also significantly suppressed by transfection with shTEM8 compared with that in the control group (Fig. 1B). These results suggested that shTEM8 transfection could successfully knock down TEM8 expression in XWLC-05 cells.

TEM8 knockdown inhibits tumor growth in a xenograft mouse model. A previous study reported that TEM8 expression was significantly increased in human lung cancer tissues compared with that in the normal tissue (11). To investigate whether TEM 8 knockdown has any influence on lung cancer cell tumorgenicity in vivo, shTEM8-transfected, shNC-transfected or untreated XWLC-05 cells were subcutaneously injected into nude mice. As shown in Fig. 2A, tumor growth was significantly decreased in the shTEM8 group compared with that in the shNC and control groups. Both final tumor size (Fig. 2B and C) and weight (Fig. 2D) were significantly decreased in the shTEM8 group compared with those in the shNC and control groups. Histological analysis was also performed to macroscopically analyze tumor growth after TEM8 expression was knocked down. The results showed that the number of tumor cells, vascular tissues and necrotic foci in the tumor tissue of shNC and control groups were markedly decreased compared with those in the shTEM8 group (Fig. 2E). Knock down of TEM8 was verified by immunohistochemistry. Tumors formed by XWLC-05 cells transfected with shTEM8 exhibited notably lower TEM8 expression compared with those in the shNC and control groups (Fig. 2F). These results suggested that TEM8 knockdown could significantly inhibit lung cancer tumor growth in vivo.

TEM8 knockdown attenuates tumor angiogenesis in mouse tumor tissues. Previous studies have reported that tumor growth is associated with angiogenesis $(23,24)$. To determine if TEM8 knockdown affects angiogenesis in vivo, the expression of the endothelial surface marker CD34 was detected by immunofluorescence staining using an anti-CD34 antibody. Compared with that in the control (Fig. 3A) and shNC groups (Fig. 3B), the expression of CD34 was notably decreased in the shTEM8 group (Fig. 3C). The MVD of tumor-bearing tissues was significantly decreased in the shTEM8 group compared with that in the shNC and control groups (Fig. 3D). Therefore, TEM8 knockdown could potently suppress angiogenesis in this xenograft tumor model.

TEM8 knockdown reduces ERK1/2 activation and Bcl-2 expression in XWLC-05 cells. A previous study reported that the ERK signaling pathway serves an important role in the 

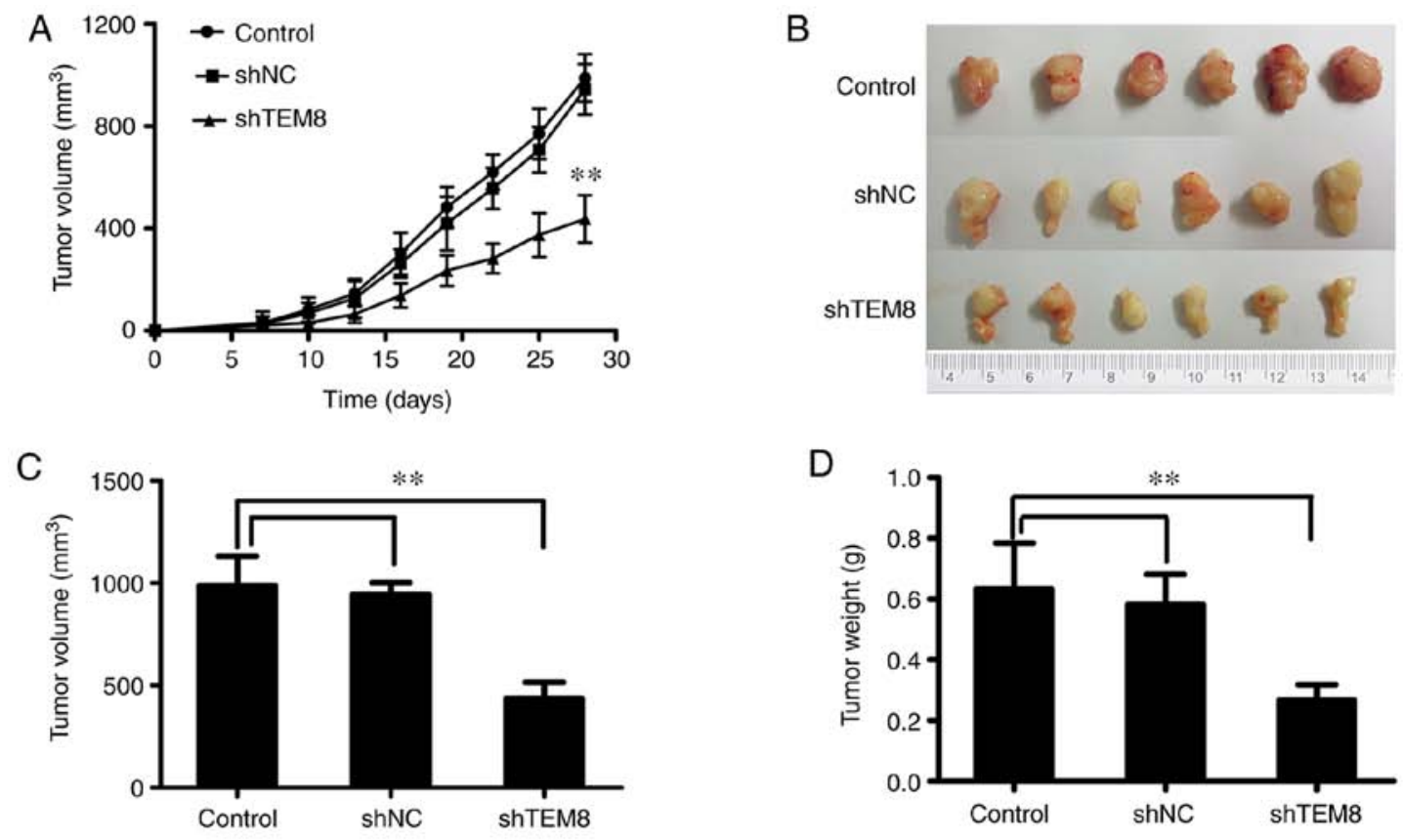

$\mathrm{E}$
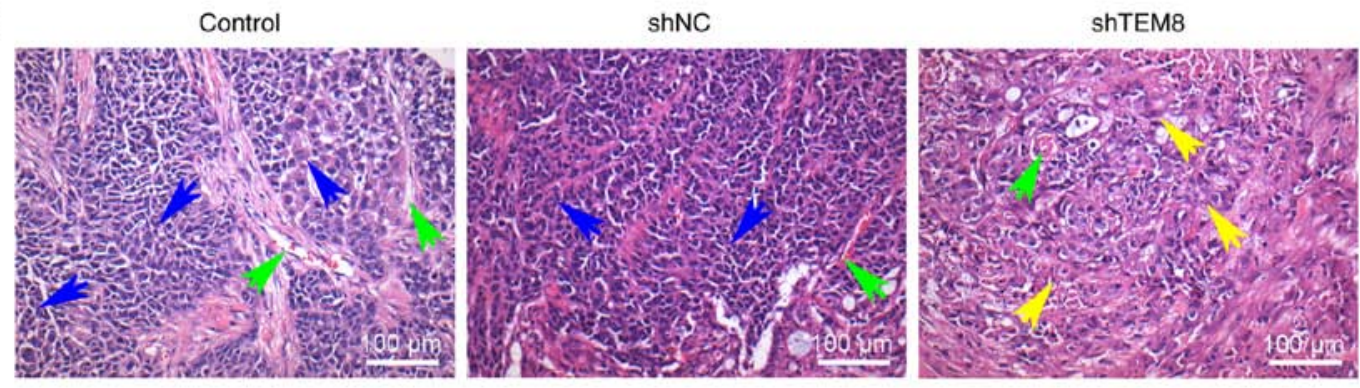

$\mathrm{F}$
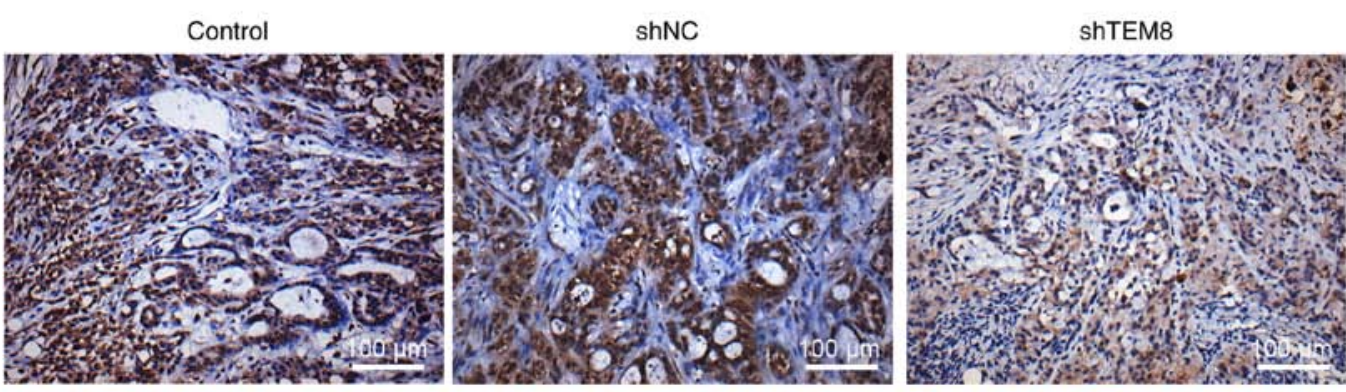

Figure 2. Effect of TEM8 silencing on tumor growth in a murine xenograft model of lung cancer in vivo. (A) Silencing TEM8 with shTEM8 significantly inhibited tumor growth of Xuanwei Lung Cancer- 05 cells implanted subcutaneously in BALB/c nude mice compared with control group, and significantly reduced (B and C) tumor volume and (D) weight. (E) Histological analysis of tumor tissues as detected by hematoxylin and eosin staining are shown. Blue arrows indicate tumor cells, green arrows indicate vascular tissues and yellow arrows indicate necrotic foci. (F) Histological features of tumor tissues by TEM8 immunohistochemistry staining. shTEM8 group tumors displayed markedly lower TEM8 expression compared with the control group. Magnification, x200. ${ }^{* *} \mathrm{P}<0.01$ vs. Control group. TEM8, tumor endothelial marker 8; sh, short hairpin RNA; NC, negative control.

pathophysiology of lung cancer (25). However, the effect of TEM8 on the ERK signaling pathway in XWLC-05 cells remains unknown. Therefore, the mRNA and protein expression levels of ERK1/2 and Bcl-2 were evaluated by RT-qPCR and western blotting at the cellular level after TEM8 gene silencing. RT-qPCR results showed that the mRNA levels of ERK and Bcl-2 were significantly decreased in shTEM8-transfected XWLC-05 cells compared with those in shNC or control XWLC-05 cells (Fig. 4A). In addition, western blot analysis showed that the protein expression levels of $\mathrm{p}$-ERK $1 / 2$ and Bcl-2 were significantly decreased in shTEM8-transfected cells compared with those in shNC or control XWLC-05 cells, although there were no variations in total ERK1/2 expression (Fig. 4B and C).

TEM8 knockdown reduces the phosphorylation of ERK1/2 and Bcl-2 expression in xenograft tumor tissues. To evaluate further if TEM8 regulates the ERK signaling pathway, the mRNA and protein levels of ERK1/2 and Bcl-2 in isolated xenograft tumor tissues were measured by RT-qPCR and western blotting. RT-qPCR results showed that the mRNA expression levels of ERK and Bcl-2 were significantly decreased in the shTEM8 group compared with that in the shNC and control groups (Fig. 5A). Moreover, western blotting analysis results revealed 
A

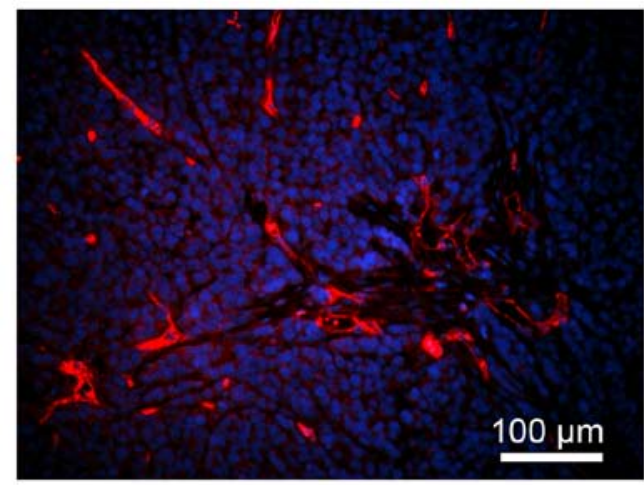

C

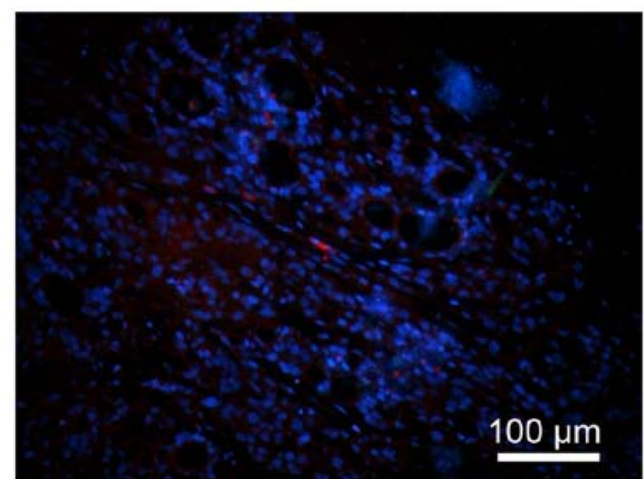

B

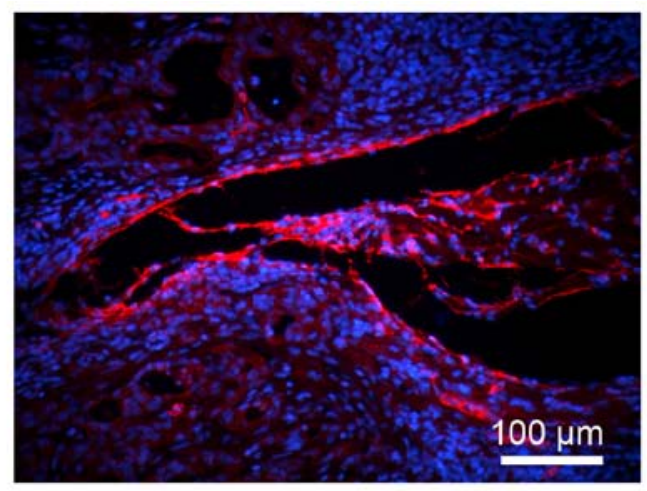

D

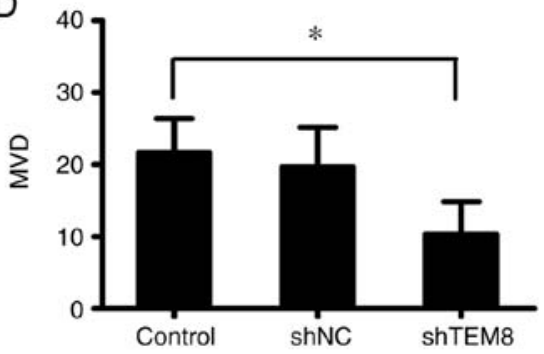

Figure 3. Effect of TEM8 silencing on tumor angiogenesis in a murine xenograft model of lung cancer in vivo. The tumor angiogenesis of the (A) control group, (B) shNC group and (C) shTEM8 group. Magnification, x200. (D) MVD. "P<0.05 vs. Control group. TEM8, tumor endothelial marker 8; sh, short hairpin RNA; $\mathrm{NC}$, negative control; MVD, microvascular density.
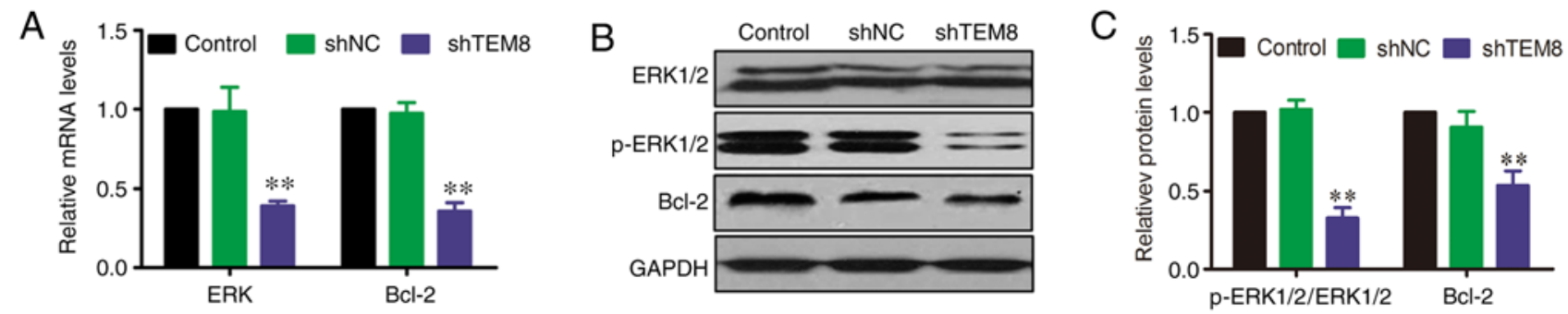

Figure 4. Silencing TEM8 significantly suppresses the activation of ERK1/2, and the expression of Bcl-2 in XWLC-05 cells. TEM8 knockdown significantly inhibited the (A) mRNA and (B and C) protein levels of ERK1/2, p-ERK1/2 and Bcl-2 in XWLC-05 cells. ${ }^{* *} \mathrm{P}<0.01$ vs. Control group. TEM8, tumor endothelial marker 8; sh, short hairpin RNA; NC, negative control; ERK, extracellular signal-regulated kinase; XWLC-05, Xuanwei Lung Cancer-05; p-, phosphorylated.

that the protein expression levels of p-ERK1/2 and Bcl-2 were significantly decreased in the shTEM8 group compared with those in the shNC and control groups (Fig. 5B and C).

\section{Discussion}

The present study sought to investigate the effects of TEM8 in a murine xenograft model of lung cancer and its underlying mechanisms. It was found that: i) TEM8 knockdown significantly inhibited tumor growth in a murine xenograft model of lung cancer; ii) TEM8 knockdown prominently attenuated tumor angiogenesis in tumor tissues of the lung cancer xenografts; and iii) TEM8 regulation of tumor growth and angiogenesis was in part mediated by activation of the ERK/Bcl-2 signaling pathway.

Previous studies have reported that TEM8 serves an important role in lung cancer carcinogenesis $(9,11,15)$, which is also important for tumor progression and growth (26). In the present study, XWLC-05 cells were used to induce stable knock down of TEM8 expression in vitro. Lung cancer in Xuanwei Country (Yunnan province, China) has been previously demonstrated to be a histological subtype of NSCLC (27). The XWCL-05 cell line established by Yan et al (16) as a human lung adenocarcinoma cell line possesses the characteristics of lung adenocarcinoma. In the present study, TEM8 knockdown was found to significantly inhibit tumor growth in a murine xenograft model of lung cancer induced by injection with the XWLC-05 cell line, which is consistent with previously reported preclinical cancer models, including colon cancer, melanoma tumors and infantile hemangioma (28-30). A number of studies have demonstrated that TEM8 is highly expressed in tumor tissues, including colon cancer, osteosarcoma and breast cancer, where it participates in tumor angiogenesis $(10,31,32)$. Importantly, a previous study also found that TEM8 is highly 
A

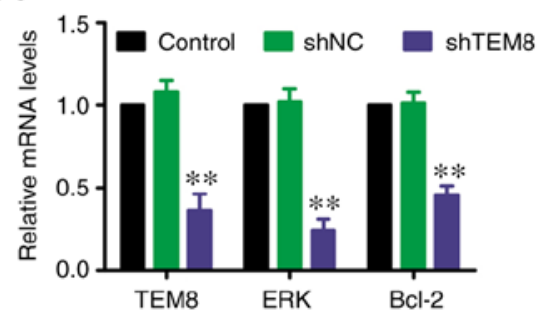

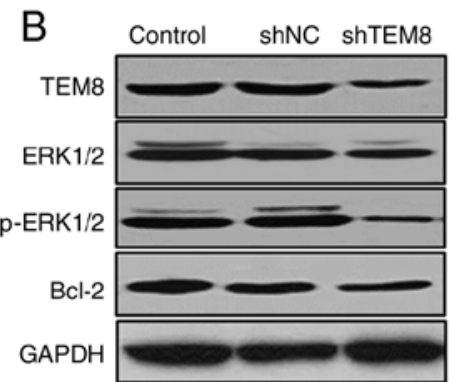

C

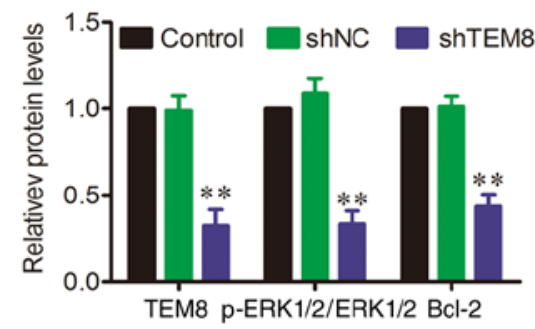

Figure 5. Silencing TEM8 significantly suppresses the activation of ERK1/2, and the expression Bcl-2 in lung cancer xenograft tumor tissues. Knockdown of TEM8 significantly inhibited the (A) mRNA levels and (B and C) protein levels of TEM8, ERK1/2, p-ERK1/2 and Bcl-2 in xenograft tumor tissues. ${ }^{* *} \mathrm{P}<0.01$ vs. Control group. TEM8, tumor endothelial marker 8; sh, short hairpin RNA; NC, negative control; ERK, extracellular signal-regulated kinase; p-, phosphorylated.

expressed in lung cancer tumor tissues compared with adjacent normal lung tissues (11). Therefore, it was hypothesized that the significantly inhibited tumor growth in mice injected with TEM8-knockdown lung cancer cells compared with that in control mice was associated with tumor angiogenesis. CD34 is a capillary endothelial cell marker, which has been used as an angiogenesis marker in previous studies (33-35) and to measure the intensity of the microvasculature (36). CD34 staining was therefore used as an angiogenesis marker in the present study to evaluate if TEM8 knockdown could inhibit tumor angiogenesis in the lung cancer tumor tissues. TEM8 knockdown was found to significantly inhibit tumor angiogenesis in tumor tissues, which was in consistent with previous findings $(14,15)$.

Although previous studies have confirmed that TEM8 knockdown can significantly inhibit tumor growth in numerous cancer types $(10,37)$, the underlying mechanism remains poorly understood. TEM8 antibodies have been reported to reduce tumor growth in mice through an antibody-dependent cellular cytotoxicity mechanism (15). The ERK signaling pathway was previously shown to participate in the progression and metastasis of lung cancer (38), where ERK1/2 phosphorylation can promote epithelial-to-mesenchymal transition and treatment resistance in lung cancer (39). ERK1/2 activity has been demonstrated to serve an important role in tumorigenesis by regulating a variety of biological processes, including apoptosis, proliferation and migration $(40,41)$. The Bcl-2 family of proteins are central regulators of apoptosis, which are traditionally divided into two types, pro-apoptotic or anti-apoptotic, where Bcl-2 is the prototypic anti-apoptotic protein (42). Several studies have previously knocked down Bcl-2 expression to evaluate anticancer efficacy in different cancer types $(43,44)$. ERK signaling has also been shown to positively regulate $\mathrm{Bcl}-2$ expression in human pancreatic cancer. (45). Blocking the ERK signaling pathway has been demonstrated to exert different outcomes on Bcl-2 expression $(42,45)$. Chang et al $(46)$ previously reported that inhibition of ERK did not affect the expression of Bcl-2 in lung cancer. Although ERK may be involved in the dysregulation of Bcl-2 expression, little is known concerning the role of the ERK signaling pathway in the regulation of tumor growth in lung cancer after TEM 8 knockdown. To investigate the expression statuses of ERK and Bcl-2 underlying the regulation of lung cancer tumor growth and angiogenesis by TEM8, RT-qPCR and western blotting were performed. TEM8 knockdown was shown to significantly inhibit ERK phosphorylation and Bcl-2 expression at both the transcriptional and protein levels. These results suggested that TEM 8 knockdown in tumor tissues significantly inhibited the ERK/Bcl-2 signaling pathway.

The present study had some limitations. For example, different lung cancer cell lines were not used to verify the results, and ERK gene interference was not performed to verify the role of TEM8 in regulating NSCLCs via the ERK/Bcl-2 signaling pathway. The occurrence and development of NSCLC is a complex biological process involving multiple signaling pathways; however, the present study only focused on the ERK signaling pathway. Therefore, further studies are required.

In conclusion, the present study suggested that TEM8 serves an important role in human lung cancer, as it was found that silencing of TEM8 expression could inhibit xenograft tumor growth of lung cancer by suppressing the ERK/Bcl-2 signaling pathways. Therefore, targeting TEM8-mediated molecular mechanisms may prove to be an effective therapeutic strategy for patients with NSCLC.

\section{Acknowledgements}

Not applicable.

\section{Funding}

The present study was supported by the Basic Research Program of Yunnan Province-Joint Project of Kunming Medical University (grant no. 2018FE001-255) and Health Science and Technology Project of Yunnan Province (grant nos. 2017NS174 and 2018NS0049).

\section{Availability of data and materials}

The datasets used and/or analyzed during the current study are available from the corresponding author on reasonable request.

\section{Authors' contributions}

LZ participated in the study design and revised the manuscript. QG and JD performed the experiments and were responsible for writing the manuscript. $\mathrm{LZ}$ and $\mathrm{CZ}$ analyzed the data. $\mathrm{CF}$ 
and XW participated in statistical analysis and organized the figures. QG and LZ confirm the authenticity of all the raw data. All authors read and approved the final manuscript.

\section{Ethics approval and consent to participate}

The experimental protocols used in the present study were approved by the Committee on the Ethics of Animal Experiments of Third Affiliated Hospital of Kunming Medical University (approval no. 16KY-LA00135).

\section{Patient consent for publication}

Not applicable.

\section{Competing interests}

The authors declare that they have no competing interests.

\section{References}

1. Lancet T: Lung cancer: Some progress, but still a lot more to do. Lancet 394: 1880, 2019.

2. Herbst RS, Heymach JV and Lippman SM: Lung cancer. N Engl J Med 359: 1367-1380, 2008.

3. Bordoloi D, Banik K, Padmavathi G, Vikkurthi R, Harsha C, Roy NK, Singh AK, Monisha J, Wang H, Kumar AP and Kunnumakkara AB: TIPE2 induced the proliferation, survival, and migration of lung cancer cells through modulation of $\mathrm{Akt} / \mathrm{mTOR} / \mathrm{NF}-\mathrm{\kappa B}$ signaling cascade. Biomolecules 9: 836, 2019.

4. Siegel RL, Miller KD and Jemal A: Cancer statistics, 2019. CA Cancer J Clin 69: 7-34, 2019.

5. Cohen JD, Li L, Wang Y, Thoburn C, Afsari B, Danilova L, Douville C, Javed AA, Wong F, Mattox A, et al: Detection and localization of surgically resectable cancers with a multi-analyte blood test. Science 359: 926-930, 2018.

6. Didkowska J, Wojciechowska U, Mańczuk M and Łobaszewski J: Lung cancer epidemiology: Contemporary and future challenges worldwide. Ann Transl Med 4: 150, 2016.

7. Martin P and Leighl NB: Review of the use of pretest probability for molecular testing in non-small cell lung cancer and overview of new mutations that may affect clinical practice. Ther Adv Med Oncol 9: 405-414, 2017.

8. Garmendia I, Pajares MJ, Hermida-Prado F, Ajona D, Bértolo C, Sainz C, Lavín A, Remírez AB, Valencia K, Moreno H, et al: YES1 drives lung cancer growth and progression and predicts sensitivity to dasatinib. Am J Respir Crit Care Med 200: 888-899, 2019.

9. St Croix B, Rago C, Velculescu V, Traverso G, Romans KE, Montgomery E, Lal A, Riggins GJ, Lengauer C, Vogelstein B and Kinzler KW: Genes expressed in human tumor endothelium. Science 289: 1197-1202, 2000.

10. Cao C, Wang Z, Huang L, Bai L, Wang Y, Liang Y, Dou C and Wang L: Down-regulation of tumor endothelial marker 8 suppresses cell proliferation mediated by ERK1/2 activity. Sci Rep 6: 23419, 2016.

11. Gong Q, Liu C, Wang C, Zhuang L, Zhang L and Wang X: Effect of silencing TEM8 gene on proliferation, apoptosis, migration and invasion of XWLC-05 lung cancer cells. Mol Med Rep 17: 911-917, 2018.

12. Koo HM, VanBrocklin M, McWilliams MJ, Leppla SH, Duesbery NS and Vande Woude GF: Apoptosis and melanogenesis in human melanoma cells induced by anthrax lethal factor inactivation of mitogen-activated protein kinase kinase. Proc Natl Acad Sci USA 99: 3052-3057, 2002.

13. Byrd TT, Fousek K, Pignata A, Szot C, Samaha H, Seaman S, Dobrolecki L, Salsman VS, Oo HZ, Bielamowicz K, et al: TEM8/ANTXR1-Specific CAR T cells as a targeted therapy for triple-negative breast cancer. Cancer Res 78: 489-500, 2018.

14. Fernando $\mathrm{S}$ and Fletcher BS: Targeting tumor endothelial marker 8 in the tumor vasculature of colorectal carcinomas in mice. Cancer Res 69: 5126-5132, 2009.
15. Chaudhary A, Hilton MB, Seaman S, Haines DC, Stevenson S, Lemotte PK, Tschantz WR, Zhang XM, Saha S, Fleming T and St Croix B: TEM8/ANTXR1 blockade inhibits pathological angiogenesis and potentiates tumoricidal responses against multiple cancer types. Cancer Cell 21: 212-226, 2012.

16. Yan FC, Wang QQ, Ruan YH, Ma LJ, Jia JT and Jin KW: Establishment and biological characteristics of lung cancer cell line XWLC-05. Ai Zheng 26: 21-25, 2007 (In Chinese).

17. Carmeliet $P$ and Jain RK: Angiogenesis in cancer and other diseases. Nature 407: 249-257, 2000.

18. Hurwitz H, Fehrenbacher L, Novotny W, Cartwright T, Hainsworth J, Heim W, Berlin J, Baron A, Griffing S, Holmgren E, et al: Bevacizumab plus irinotecan, fluorouracil, and leucovorin for metastatic colorectal cancer. N Engl J Med 350: 2335-2342, 2004.

19. Verheul HM and Pinedo HM: Possible molecular mechanisms involved in the toxicity of angiogenesis inhibition. Nat Rev Cancer 7: 475-485, 2007.

20. Chen HX and Cleck JN: Adverse effects of anticancer agents that target the VEGF pathway. Nat Rev Clin Oncol 6: 465-477, 2009.

21. Livak KJ and Schmittgen TD: Analysis of relative gene expression data using real-time quantitative PCR and the 2(-Delta Delta C(T)) method. Methods 25: 402-408, 2001.

22. Weidner N: Current pathologic methods for measuring intratumoral microvessel density within breast carcinoma and other solid tumors. Breast Cancer Res Treat 36: 169-180, 1995.

23. Hernandez JL, Padilla L, Dakhel S, Coll T, Hervas R, Adan J, Masa M, Mitjans F, Martinez JM, Coma S, et al: Therapeutic targeting of tumor growth and angiogenesis with a novel Anti-S100A4 monoclonal antibody. PLoS One 8: e72480, 2013.

24. Craig M, Ying C and Loberg RD: Co-inoculation of prostate cancer cells with U937 enhances tumor growth and angiogenesis in vivo. J Cell Biochem 103: 1-8, 2008.

25. Lu Z, Ding L, Hong H, Hoggard J, Lu Q and Chen YH: Claudin-7 inhibits human lung cancer cell migration and invasion through ERK/MAPK signaling pathway. Exp Cell Res 317: 1935-1946, 2011.

26. Kerbel RS: Tumor angiogenesis. N Engl J Med 358: 2039-2049, 2008.

27. Li R, Liu Y, Wang T, Tang J, Xie L, Yao Z, Li K, Liao Y, Zhou L, Geng Z, et al: The characteristics of lung cancer in Xuanwei County: A review of differentially expressed genes and noncoding RNAs on cell proliferation and migration. Biomed Pharmacother 119: 109312, 2019.

28. Jinnin M, Medici D, Park L, Limaye N, Liu Y, Boscolo E, Bischoff J, Vikkula M, Boye E and Olsen BR: Suppressed NFAT-dependent VEGFR1 expression and constitutive VEGFR2 signaling in infantile hemangioma. Nat Med 14: 1236-1246, 2008.

29. Carson-Walter EB, Watkins DN, Nanda A, Vogelstein B, Kinzler KW and St Croix B: Cell surface tumor endothelial markers are conserved in mice and humans. Cancer Res 61: 6649-6655, 2001.

30. Nanda A, Carson-Walter EB, Seaman S, Barber TD, Stampfl J, Singh S, Vogelstein B, Kinzler KW and St Croix B: TEM8 interacts with the cleaved C5 domain of collagen alpha 3(VI). Cancer Res 64: 817-820, 2004.

31. Rmali KA, Watkins G, Harrison G, Parr C, Puntis MC and Jiang WG: Tumour endothelial marker 8 (TEM-8) in human colon cancer and its association with tumour progression. Eur J Surg Oncol 30: 948-953, 2004.

32. Chen D, Bhat-Nakshatri P, Goswami C, Badve S and Nakshatri H: ANTXR1, a stem cell-enriched functional biomarker, connects collagen signaling to cancer stem-like cells and metastasis in breast cancer. Cancer Res 73: 5821-5833, 2013.

33. Popravka ES, Dyatlova AS, Lin'kova NS, Krylova YS Polyakova VO and Kvetnoi IM: Role of LIF Cytokine and CD34 angiogenesis marker in non-developing pregnancy. Bull Exp Biol Med 163: 772-776, 2017.

34. Vieira SC, Silva BB, Pinto GA, Vassallo J, Moraes NG, Santana JO, Santos LG, Carvasan GA and Zeferino LC: CD34 as a marker for evaluating angiogenesis in cervical cancer. Pathol Res Pract 201: 313-318, 2005.

35. Tanaka F, Otake Y, Yanagihara K, Kawano Y, Miyahara R, Li M, Ishikawa $\mathrm{S}$ and Wada H: Correlation between apoptotic index and angiogenesis in non-small cell lung cancer: Comparison between CD105 and CD34 as a marker of angiogenesis. Lung Cancer 39: 289-296, 2003. 
36. Ceyran AB, Senol S, Güzelmeriç F, Tuncer E, Tongut A, Özbek B, Şavluk Ö, Aydın A and Ceyran H: Effects of hypoxia and its relationship with apoptosis, stem cells, and angiogenesis on the thymus of children with congenital heart defects: A morphological and immunohistochemical study. Int J Clin Exp Pathol 8: 8038-8047, 2015.

37. Opoku-Darko M, Yuen C, Gratton K, Sampson E and Bathe OF: Tumor endothelial marker 8 overexpression in breast cancer cells enhances tumor growth and metastasis. Cancer Invest 29: 676-682, 2011.

38. Heigener DF, Gandara DR and Reck M: Targeting of MEK in lung cancer therapeutics. Lancet Respir Med 3: 319-327, 2015.

39. Kim E, Youn H, Kwon T, Son B, Kang J, Yang HJ, Seong KM, Kim W and Youn B: PAK1 tyrosine phosphorylation is required to induce epithelial-mesenchymal transition and radioresistance in lung cancer cells. Cancer Res 74: 5520-5531, 2014.

40. Chang CH, Li JR, Shu KH, Fu YC and Wu MJ: Hydronephrotic urine in the obstructed kidney promotes urothelial carcinoma cell proliferation, migration, invasion through the activation of mTORC2-AKT and ERK signaling pathways. PLoS One 8 e74300, 2013.

41. Koyama T, Ogawara K, Kasamatsu A, Okamoto A, Kasama H, Minakawa $Y$, Shimada $K$, Yokoe $H$, Shiiba M, Tanzawa $H$ and Uzawa K: ANGPTL3 is a novel biomarker as it activates ERK/MAPK pathway in oral cancer. Cancer Med 4: 759-769, 2015.

42. Galante JM, Mortenson MM, Bowles TL, Virudachalam S and Bold RJ: ERK/BCL-2 pathway in the resistance of pancreatic cancer to anoikis. J Surg Res 152: 18-25, 2009.
43. Lariche N, Lahouel M, Benguedouar L and Zellagui A: Ferulenol, a sesquiterpene coumarin, induce apoptosis via mitochondrial dysregulation in lung cancer induced by Benzo[a]pyrene: Involvement of $\mathrm{Bcl} 2$ protein. Anticancer Agents Med Chem 17: 1357-1362, 2017

44. Beider K, Begin M, Abraham M, Wald H, Weiss ID, Wald O, Pikarsky E, Zeira E, Eizenberg O, Galun E, et al: CXCR4 antagonist 4F-benzoyl-TN14003 inhibits leukemia and multiple myeloma tumor growth. Exp Hematol 39: 282-292, 2011.

45. Boucher MJ, Morisset J, Vachon PH, Reed JC, Lainé J and Rivard N: MEK/ERK signaling pathway regulates the expression of Bcl-2, Bcl-X(L), and Mcl-1 and promotes survival of human pancreatic cancer cells. J Cell Biochem 79: 355-369, 2000.

46. Chang GC, Hsu SL, Tsai JR, Wu WJ, Chen CY and Sheu GT: Extracellular signal-regulated kinase activation and Bcl-2 downregulation mediate apoptosis after gemcitabine treatment partly via a p53-independent pathway. Eur J Pharmacol 502: 169-183, 2004.

This work is licensed under a Creative Commons Attribution-NonCommercial-NoDerivatives 4.0 International (CC BY-NC-ND 4.0) License. 